\title{
Shot noise of large charge quanta in superconductor/semiconductor/superconductor junctions
}

\author{
F. E. Camino, V. V. Kuznetsov, and E. E. Mendez \\ Department of Physics and Astronomy, Stony Brook University, Stony Brook, New York 11794-3800, USA \\ Th. Schäpers, V. A. Guzenko, and H. Hardtdegen \\ Institut für Schichten und Grenzflächen (ISG-1), Forschungszentrum Jülich, D-52425 Jülich, Germany \\ (Received 25 June 2004; revised manuscript received 6 October 2004; published 28 January 2005)
}

\begin{abstract}
We have found experimentally that the noise of ballistic electron transport in a superconductor/ semiconductor/superconductor junction is enhanced relative to the value given by the general relation, $S_{V}=2 e I R^{2} \operatorname{coth}(e V / 2 k T)$, for two voltage regions in which this expression reduces to its thermal and shot noise limits. The noise enhancement is explained by the presence of large charge quanta, with an effective charge $q^{*}=(1+2 \Delta / e V) e$, that generate a noise spectrum $S_{V}=2 q^{*} I R^{2}$, as predicted by Averin and Imam [Phys. Rev. Lett. 76, 3814 (1996)]. These charge quanta result from multiple Andreev reflections at each junction interface, which are also responsible for the subharmonic gap structure observed in the voltage dependence of the junction's conductance.
\end{abstract}

DOI: 10.1103/PhysRevB.71.020506

In the last few years there has been an increasing interest in measuring shot noise since it can give a more complete picture of the physics involved in a system under study than that offered by conductance measurements alone. ${ }^{1}$ Hybrid superconductor/semiconductor/superconductor $(\mathrm{S} / \mathrm{Sm} / \mathrm{S})$ devices with few one-dimensional channels, referred to in the literature as superconducting quantum point contacts (SQPC), provide an example of the potential usefulness of shot noise in that regard. It has been predicted that in these devices the shot noise should be much larger than the Poissonian noise $S_{I}=2 e I$ generated by electrons of charge $e$, as if it were created by a large charge quantum of the order of $2 \Delta / V(e V \ll \Delta)$, where $\Delta$ is the energy gap of the superconducting electrodes and $V$ is the applied voltage across the device. $^{2}$

This large charge quantum can be seen as a consequence of a phenomenon (Andreev reflection) occurring at the $\mathrm{Sm} / \mathrm{S}$ interface when the energy, $E$, of a quasiparticle incident on the interface from the semiconductor side, is inside the energy gap of the superconducting electrode. ${ }^{3}$ Under this condition, the quasiparticle (e.g., an electron) cannot enter the superconductor and cannot be reflected from the interface (assuming an interface with zero potential height), hence, the only possibility is for the quasiparticle to annihilate, with the production of a retroreflected hole of energy $-E$ in the semiconductor side and a Cooper pair on the superconductor side (exactly the inverse occurs if a hole hits the interface).

In a SQPC with a bias $V$ across its electrodes, a quasiparticle coming from one of the electrodes generates a chain of $2 \Delta / e V(e V<2 \Delta)$ Andreev reflections, each pair of which transfers a charge $2 e$ across the junction, until the last Andreev-reflected particle is injected into a quasiparticle level in the opposite superconductor electrode. As a consequence, this chain process transfers a net large charge quantum $q^{*} \approx(1+2 \Delta / e V) e$, whose shot noise has been predicted by Averin and Imam to be $S_{V}=2 q * I R^{2}$ [Ref. 2].

Shot-noise enhancement and an indication of large charge quanta have been found experimentally in S/insulator/S tunnel junctions ${ }^{4}$ and in S/normal metal/S junctions. ${ }^{5}$ Furthermore, quantitative confirmation of the theory of shot noise in
PACS number(s): 74.40.+k, 74.45.+c, 74.50.+r

SQPC (Ref. 6) was found in aluminum point contacts, ${ }^{7}$ supporting the idea that multiple Andreev reflections are responsible for dissipative charge transfer between superconductors. There have also been reports of shot noise in semiconductor-based junctions being enhanced. For instance, the shot noise in a S/insulator/Sm junction has been found to be twice the Poissonian noise; ${ }^{8}$ and in a quasidiffusive $\mathrm{S} / \mathrm{Sm} / \mathrm{S}$ junction it has been shown that in the limit of incoherent multiple Andreev reflections, the shot noise is enhanced due to an increase in the electron temperature with respect to the lattice temperature. ${ }^{9}$ However, to the best of our knowledge, there has not been any evidence of large charge quanta in semiconductor-based junctions, probably due to the very strict demands required for that observation, namely, a large density of high-mobility electrons and a high electron transparency of the $\mathrm{Sm} / \mathrm{S}$ interfaces. ${ }^{10}$

We report here that by paying special attention to materials and device optimization we have been able to observe shot noise of large charge quanta in $\mathrm{S} / \mathrm{Sm} / \mathrm{S}$ junctions. To this effect, we have used a hybrid device that consisted of a two-dimensional electron gas (2-DEG) defined by modulation doping in an $\mathrm{In}_{0.53} \mathrm{Ga}_{0.47} \mathrm{As}_{\mathrm{s}} / \mathrm{In}_{0.77} \mathrm{Ga}_{0.23} \mathrm{As} / \mathrm{InP}$ heterostructure. ${ }^{11}$ The 2-DEG was bound laterally by two $\mathrm{Nb}$ contacts $(3 \mu \mathrm{m}$ wide) separated by a distance, $L$, of $0.4 \mu \mathrm{m}$. The 2-DEG mobility and carrier density, measured at $4.2 \mathrm{~K}$, were $3.5 \times 10^{5} \mathrm{~cm}^{2} / \mathrm{V} \mathrm{s}$ and $6.6 \times 10^{11} \mathrm{~cm}^{-2}$, respectively. As a consequence, the electronic mean free path, $l$, and coherence length, $\xi$ (at $1.2 \mathrm{~K}$ ), were 4.6 and $0.6 \mu \mathrm{m}$, respectively. Since $l$ and $\xi$ are larger than $L$, the electronic transport in our device is ballistic and the probability of sustaining multiple Andreev reflections (MARs) is high, provided that the interface has very good transparency. ${ }^{10}$ This condition was favored by confining the 2-DEG within the $\mathrm{In}_{0.77} \mathrm{Ga}_{0.23} \mathrm{As}$ layer, which itself presents a zero Schottky barrier at the lateral metal/semiconductor interfaces. ${ }^{12}$ In addition, the $\mathrm{Nb}$ electrodes were deposited with an ion beam deposition system that allowed in situ cleaning of the semiconductor lateral wall prior to the metal evaporation; this process has proven to be crucial for a good transparency of the $\mathrm{Nb} / 2$-DEG interface. ${ }^{13}$ 
The current-biased noise measurement setup was based on a commercial (Quantum Design) superconducting quantum interference device (SQUID). The voltage fluctuations across the sample were converted by the sensor resistor, $R_{\text {sensor }}=100 \Omega$, into current fluctuations that were measured by the SQUID. Ultimately, the SQUID proportionally transformed the current circulating through its input coil into a voltage with a maximum gain of $4 \times 10^{6} \mathrm{~V} / \mathrm{A}$. Analogously, in order to measure the current-voltage curves, a known dc current was injected to the sample, and the voltage drop across it was measured by the SQUID after conversion to current by $R_{\text {sensor. }}{ }^{14}$ The junction resistance $d V / d I$ was measured directly by injecting into the current leads a small ac signal of $3 \mathrm{nA}$ and $\sim 9 \mathrm{~Hz}$ through a transformer and detecting the ac response across the sample with a lock-in amplifier, after amplification by the SQUID electronics. The current leads were of the twin BNC-type, filtered at the end close to the sample with an $R C$ filter with cutoff frequency close to $10 \mathrm{~Hz}$. The filter, the sample $(R), R_{\text {sensor }}$, and the SQUID were placed close to each other, shielded with a lead casing, and inside a liquid-helium cryostat that could be pumped down to $1.2 \mathrm{~K}$. To reduce extrinsic noise during the measurements, the power supplies for the current source (voltage source followed by a large current limiting resistor) and voltmeter (used to read a voltage proportional to the dc current) were battery powered. For the same reason, the cryostat and measurement devices were placed inside an rfshielded room.

We found that the measured noise was frequency independent in the approximate range of $1-10 \mathrm{kHz}$, but increased at higher frequencies due to the effect of the SQUID's input coil impedance. The background noise of $1.3 \mathrm{pA} / \mathrm{Hz}^{1 / 2}$ at $1.2 \mathrm{~K}$, measured directly with the sample in the zeroresistance state, was current independent (tested for samples with critical currents up to $0.1 \mathrm{~mA}$ ) for temperatures below the critical temperature, as well as at $4.2 \mathrm{~K}$, with the sample in the normal state. The background noise measured at $2.6 \mathrm{~K}$ (with the sample in the zero-resistance state) was the same as that measured at $4.2 \mathrm{~K}$, which showed that the background noise was not dependent on the load resistance, as was expected since the sample resistance $(13 \Omega)$ was much smaller than $R_{\text {sensor }}$. In addition, we estimate that no significant current noise (calculated to be $0.02 \mathrm{pA} / \mathrm{Hz}^{1 / 2}$ at the sample after the cold $R C$ filters) was externally injected through the current leads by the current source. Furthermore, the thermal current noise of the $10 \mathrm{k} \Omega$ resistors that are part of the cold $R C$ filters are not taken into account since these large resistors are effectively shunted by the sample $(\sim 10 \Omega)$. Finally, the background noise at $4.2 \mathrm{~K}$ was of $1.6 \mathrm{pA} / \mathrm{Hz}^{1 / 2}$, slightly temperature dependent in the range of 1.2-4.2 K reflecting the temperature dependence of the SQUID's internal noise.

The results summarized in Figs. 1 and 2 provide evidence that in our system Andreev reflection processes are dominant, namely, the presence of excess current $\left(I_{\text {exc }}\right)$ and of subharmonic gap structure in the transport characteristics. The current-voltage curves (CVC) in Fig. 1 show a drastic change with temperature, most notably from 4.2 to $2.6 \mathrm{~K}$. While at $4.2 \mathrm{~K}$ the current is almost linear with voltage, as it corresponds to a "normal" metal (with resistance $R_{N}=13 \Omega$ ), at $2.6 \mathrm{~K}$ and below there appears a superconducting zero-

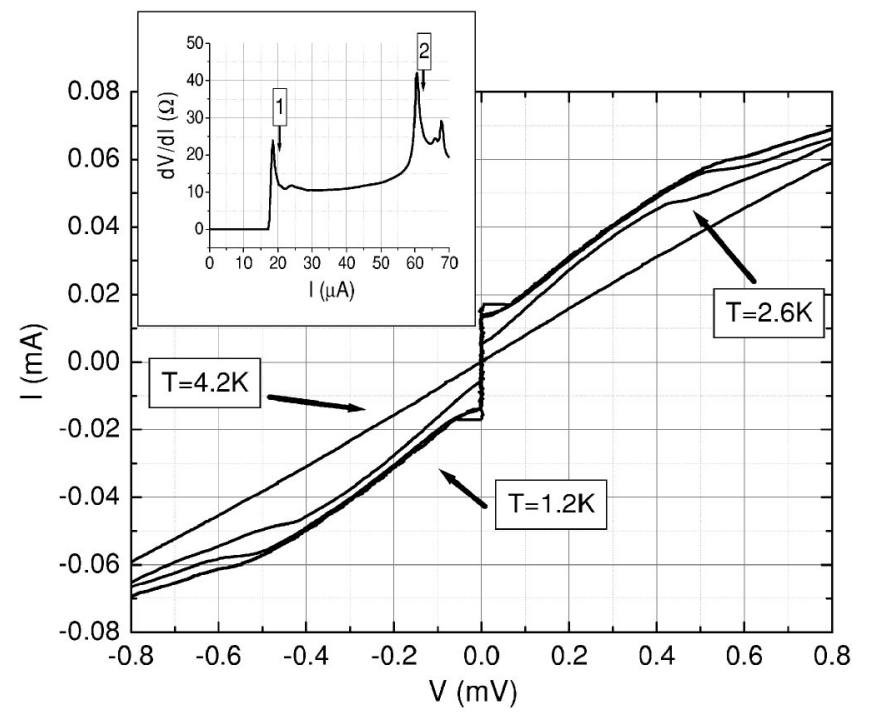

FIG. 1. Current-voltage curves for the device described in detail in the text, measured at several temperatures. Inset: The $d V / d I$ vs $V$ curve measured at $1.2 \mathrm{~K}$ corresponding to a sweep down of the current. The arrows in the inset point at the current (or voltage) regions selected for noise measurements.

resistance region at the origin, followed by the onset of finite resistance when the current exceeds the critical current $I_{c} \approx 17 \mu \mathrm{A}$. With increasing current the resistance varies and even shows some structure, as illustrated in the inset of Fig. 1. The current difference, measured in the region of large voltages, between the CVC with a superconducting state and the CVC with only a normal state is the so-called excess current; its presence in the low-temperature characteristics of Fig. 1 is a clear indication of the existence of Andreev reflections in our device. ${ }^{10}$

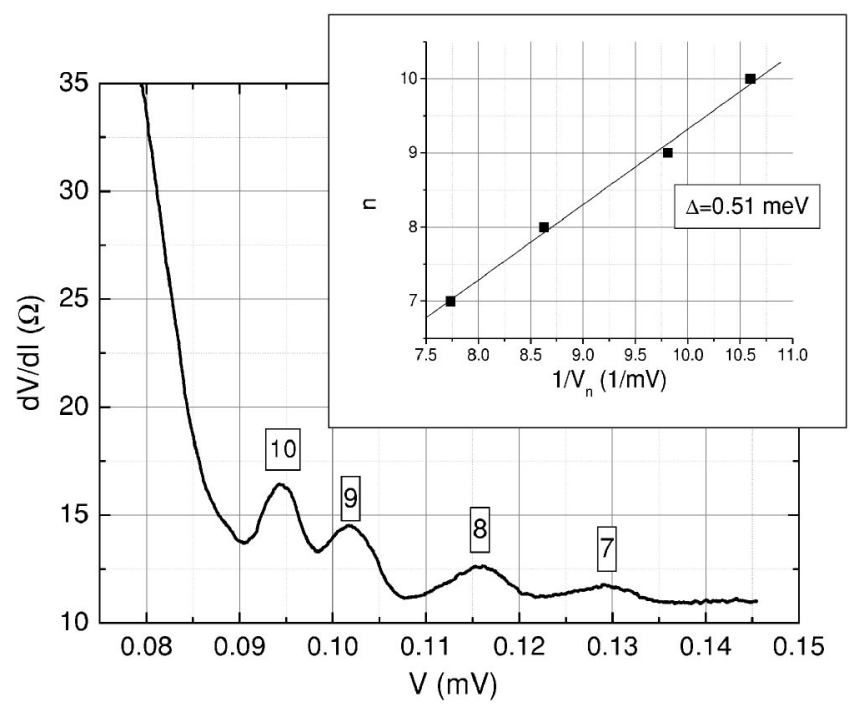

FIG. 2. Dependence of resistance on voltage in region 1 of the inset in Fig. 1, measured at $1.2 \mathrm{~K}$, for a downward sweep of the current-voltage curve. We attribute the resistance oscillations to subharmonic gap structure, from which we can determine the energy gap $\Delta$, as shown in the inset. (See the text for the method used to find $\Delta$.) The labels above the maxima of the oscillations denote the corresponding number of Andreev reflections, $n$. 
The differential resistance, $d V / d I$, measured as a function of voltage in the region near the superconducting-normal transition (region 1 in the inset of Fig. 1) exhibits oscillations, known in the literature as subharmonic gap structure (SGS), which are a consequence of multiple Andreev reflections. ${ }^{15,16}$ The maxima of the oscillations appear at voltages $V_{n}=2 \Delta / n e$, where $n$ is an integer which corresponds to the number of Andreev reflections at the $\mathrm{Sm} / \mathrm{S}$ interface, and $\Delta$ is the superconducting energy gap of the electrodes. By finding the slope in a plot of $n$ vs $1 / V_{n}$ (see inset of Fig. 2), we have obtained $\Delta=0.51 \mathrm{meV}$. The voltages of additional maxima in the device resistance, namely $V=0.6 \mathrm{mV}$ (corresponding to the peak at $I=60 \mu \mathrm{A}$ in the inset of Fig. 1) and $V=1.0 \mathrm{mV}$ (not shown), correspond, within experimental error, to the $n=2$ and $n=1$ elements, respectively, in the $V_{n}=2 \Delta / n e$ series. Since each pair of Andreev reflections in a MAR chain involves the transfer of a Cooper pair (of charge $2 e$ ) across the junction, we can express the average transferred charge, $q^{*}$, as a function of $V$ as $q^{*} \approx(1+2 \Delta / e V) e$.

At this point, several remarks are in place. First, the value of $\Delta$ determined from the SGS is considerably lower than the value of $\sim 1.5 \mathrm{meV}$ found in the literature for bulk $\mathrm{Nb}$. Although we do not have an explanation for this effect, we note that reduced values of $\Delta$ have also been found in previous works ${ }^{17}$ in addition, the product $e I_{c} R_{N}=0.22 \mathrm{meV}$ is comparable to the values we have found in similar junctions and smaller than $\Delta$ as expected for this kind of devices. ${ }^{18}$ Second, the superconducting state of the device occurred at a temperature $(<4 \mathrm{~K})$ well below the critical temperature $(7.5 \mathrm{~K})$ of the $\mathrm{Nb}$ electrodes by themselves (measured independently), which indicates the absence of electrical shorts in the semiconductor region between the electrodes. This was confirmed by inspecting the junction under a scanning electron microscope and by performing an x-ray material analysis of the interelectrode region. ${ }^{19}$ On the other hand, we observed that the critical current was approximately constant for magnetic fields up to $1 \mathrm{mT}$ (maximum attainable in our setup), and did not reveal any of the Fraunhofer-diffraction-like features expected for homogeneous transport across the whole width of the device. ${ }^{20}$ We believe that the cleaning procedure used may have created an interface with a largely inhomogeneous transparency that translates into an inhomogeneous distribution of conduction channels of small area. Our structure approaches then the SQPC regime and it therefore seems justified to interpret our experimental results in the light of the theory described in Ref. 2.

The voltage noise measured as a function of current is shown in Figs. 3 and 4, for the two regions of current labeled 1 and 2 in the inset of Fig. 1 and measured at $1.2 \mathrm{~K}$ and at a frequency of $3 \mathrm{kHz}$. Regions 1 and 2 in that inset correspond to the thermal and shot-noise limits, respectively, of the wellestablished general relation for the dependence of noise on $V$ and temperature ${ }^{21}$

$$
S_{V}=2 e I R^{2} \operatorname{coth}(e V / 2 k T),
$$

in which the crossover from thermal $\left(S_{V}=4 k T I R^{2} / V\right)$ to shot noise $\left(S_{V}=2 e I R^{2}\right)$ occurs at around $e V=2 k T$ (see Ref. 22). In both regions, the measured noise (thick solid lines in Figs. 3

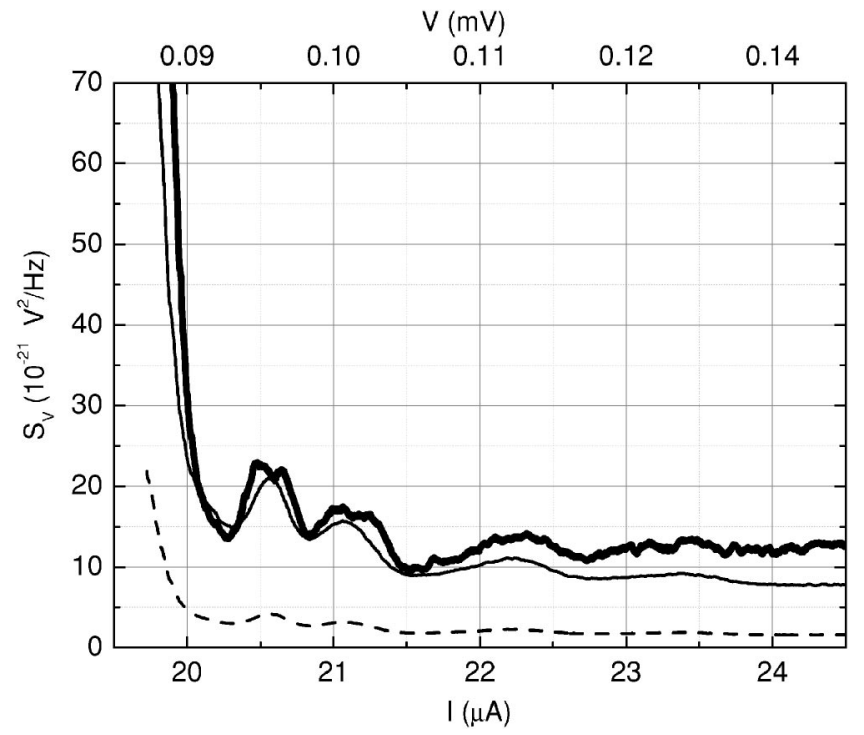

FIG. 3. Dependence of noise on voltage for region 1 (see the inset in Fig. 1), measured at $1.2 \mathrm{~K}$, corresponding to a downward sweep of the current in the hysteretic current-voltage curve. The thick solid line is the experimental curve, while the dashed and thin solid lines correspond to plots using Eq. (1) and Eq. (2), respectively. The value of $\Delta=0.51 \mathrm{meV}$ used in Eq. (2) was found from the subharmonic gap structure (see text and Fig. 2).

and 4) is significantly larger than that predicted theoretically (dashed lines) for the two limits of Eq. (1), with enhancement factors of approximately 6 and 3, for regions 1 and 2, respectively.

Since our device unambiguously presents the signatures of multiple Andreev reflections, as described above, we have interpreted the enhanced noise as the shot noise of an effective charge, $q^{*}$, along similar lines to those followed in S/insulator/S junctions. ${ }^{4}$ In Eq. (1) we then replace

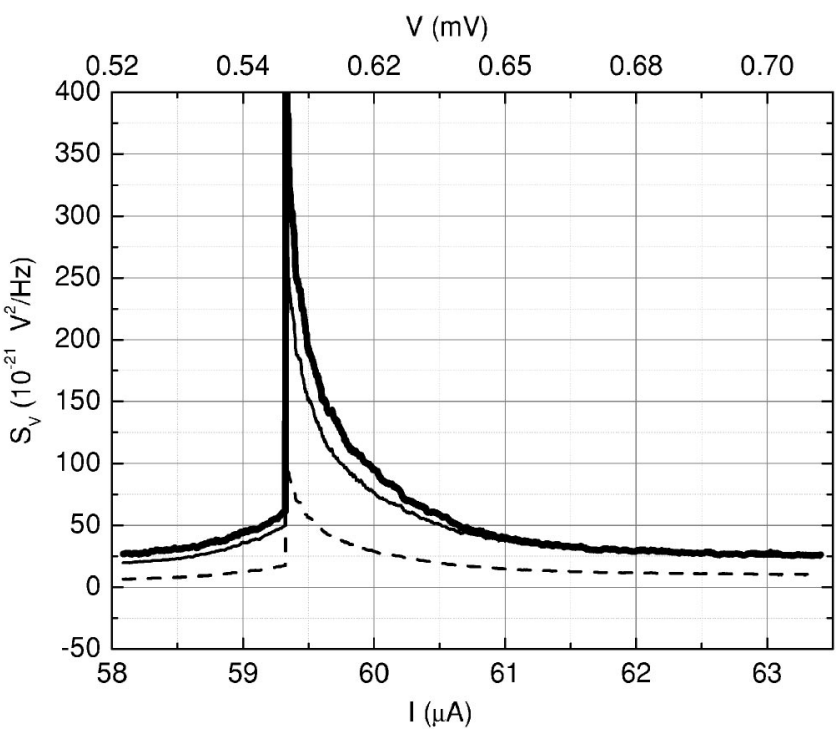

FIG. 4. Dependence of noise on voltage for region 2 (with the same considerations as for Fig. 3). We observe that Eq. (2), with the measured value of $\Delta$, reproduces satisfactorily the measured curve without the need of any fitting parameter. 
the electron charge, $e$, by the average transferred charge $q^{*}=(1+2 \Delta / e V) e$. After this substitution and using the value of $\Delta=0.51 \mathrm{meV}$ mentioned above, the $\operatorname{coth}\left(q^{*} V / 2 \mathrm{kT}\right)$ factor becomes approximately one for the two current (or voltage) ranges considered here. Consequently, the measured noise can be approximated by the expression

$$
S_{V}=2 q * I R^{2},
$$

where the effective charge depends on voltage.

To test this analysis, we have plotted in Figs. 3 and 4 (thin solid lines), the dependence of voltage noise on current calculated using Eq. (2). As shown there, the agreement with the measured values is very good throughout both regions, and justifies our explanation of noise in terms of an effective charge different from the electron charge.

The observation of enhanced shot noise in a $\mathrm{S} / \mathrm{Sm} / \mathrm{S}$ junction due to large charge quanta opens the door to the study of shot noise in other configurations in which Andreev reflections remain the main mechanism for electronic transport. For instance, by adding a split gate to the configuration studied here, it would be possible to electrostatically tune in a continuous way the number of conduction channels and systematically test the predictions for shot noise in S/Normal/S junctions, from the single mode to the multimode regime. ${ }^{6,7}$ It would also be interesting to measure the shot noise of
$\mathrm{S} / \mathrm{Sm} / \mathrm{S}$ junctions with hot carriers injected through separate electrodes. Since the supercurrent in a multiterminal $\mathrm{S} / \mathrm{Sm} / \mathrm{S}$ junction can be controlled by the injection of hot carriers, ${ }^{23}$ it is reasonable to speculate that the electronic noise might be affected as well, maybe reflecting a new effective electronic temperature induced by the hot injection.

In conclusion, we have measured electron noise in a ballistic superconductor/semiconductor/superconductor junction, and found it to be enhanced with respect to the value given by the general relation, $S_{V}=2 e I R^{2} \operatorname{coth}(e V / 2 k T)$, for two voltage regions in which this expression reverts to its thermal and shot noise limits. Additionally, we have found that we can explain the measured noise if we consider it as the shot noise, $S_{V}=2 q^{*} I R^{2}$, of an effective charge $q^{*}=(1$ $+2 \Delta / e V) e$, as predicted by theory. ${ }^{2}$ These large charge quanta result from the multiple Andreev reflection process responsible for the subharmonic gap structure that we have observed in the $d V / d I$ vs $V$ curve, and from which we have determined the value of $\Delta$ used in the expression for $q^{*}$.

We would like to thank Professor Richard Gambino and Professor James Lukens from Stony Brook University for letting us use their laboratory facilities in the course of this work. This investigation has been sponsored by the National Science Foundation under Grant No. DMR-0305384.
${ }^{1}$ For a comprehensive review of shot noise in mesoscopic devices, see, e.g., Ya. M. Blanter and M. Büttiker, Phys. Rep. 336, 1 (2000).

${ }^{2}$ D. Averin and H. T. Imam, Phys. Rev. Lett. 76, 3814 (1996).

${ }^{3}$ A. F. Andreev, Sov. Phys. JETP 19, 1228 (1964).

${ }^{4}$ P. Dieleman, H. G. Bukkems, T. M. Klapwijk, M. Schicke, and K. H. Gundlach, Phys. Rev. Lett. 79, 3486 (1997).

${ }^{5}$ T. Hoss, C. Strunk, T. Nussbaumer, R. Huber, U. Staufer, and C. Schönenberger, Phys. Rev. B 62, 4079 (2000).

${ }^{6}$ J. C. Cuevas, A. Martín-Rodero, and A. Levy Yeyati, Phys. Rev. Lett. 82, 4086 (1999); Y. Naveh and D. V. Averin, ibid. 82, 4090 (1999).

${ }^{7}$ R. Cron, M. F. Goffman, D. Esteve, and C. Urbina, Phys. Rev. Lett. 86, 4104 (2001).

${ }^{8}$ F. Lefloch, C. Hoffmann, M. Sanquer, and D. Quirion, Phys. Rev. Lett. 90, 067002 (2003).

${ }^{9}$ P. Roche, H. Perrin, D. C. Glattli, H. Takayanagi, and T. Akazaki, Physica C 352, 73 (2001).

${ }^{10}$ G. E. Blonder, M. Tinkham, and T. M. Klapwijk, Phys. Rev. B 25, 4515 (1982).

${ }^{11}$ For information about the growth of this heterostructure system, see, e.g., H. Hardtdegen, R. Meyer, M. Hollfelder, Th. Schäpers, J. Appenzeller, H. Løken-Larsen, Th. Klocke, Christel Dieker, B. Lengeler, and H. Lüth, J. Appl. Phys. 73, 4489 (1993).

${ }^{12}$ C. A. Mead and W. G. Spitzer, Phys. Rev. 134, 713 (1964); J. N. Walpole and K. W. Nill, J. Appl. Phys. 42, 5609 (1971).

${ }^{13}$ For a review of the subject, see, e.g., T. Schäpers, Superconductor/Semiconductor Junctions (Springer-Verlag, Berlin, 2001), Chap. 4.
${ }^{14}$ Since the junction lies in parallel with the series combination of the SQUID's input coil and $R_{\text {sensor }}$, the junction current is calculated by subtracting the measured current through the SQUID's input coil from the known injected current to the circuit.

${ }^{15}$ T. M. Klapwijk, G. E. Blonder, and M. Tinkham, Physica B \& C 110, 1657 (1982); M. Octavio, M. Tinkham, G. E. Blonder, and T. M. Klapwijk, Phys. Rev. B 27, 6739 (1983).

${ }^{16}$ E. N. Bratus, V. S. Shumeiko, and G. Wendin, Phys. Rev. Lett. 74, 2110 (1995); D. Averin and A. Bardas, ibid. 75, 1831 (1995); J. C. Cuevas, A. Martín-Rodero, and A. Levy Yeyati, Phys. Rev. B 54, 7366 (1996).

${ }^{17}$ See, e. g., C. Nguyen, Ph. D. thesis, University of California at Santa Barbara, 1993; A. Chrestin, T. Matsuyama, and U. Merkt, Phys. Rev. B 55, 8457 (1997).

${ }^{18}$ The maximum value for $e I_{c} R_{N}$ in an ideal ballistic $\mathrm{S} /$ constriction/S junction is $(4 / \pi) \Delta$ as derived by I. O. Kulik and A. N. Omelyanchuk, Sov. J. Low Temp. Phys. 3, 459 (1977).

${ }^{19}$ F. E. Camino, Ph.D. thesis, Stony Brook University, 2003.

${ }^{20}$ See, e.g., T. Schäpers, Superconductor/Semiconductor Junctions (Springer-Verlag, Berlin, 2001), Chap. 6, p. 83.

${ }^{21}$ M. Büttiker, Phys. Rev. B 46, 12485 (1992).

${ }^{22}$ In addition, the current for the two selected noise measurement regions was low enough to allow us to use the maximum gain of the SQUID electronics.

${ }^{23}$ Th. Schäpers, V. A. Guzenko, R. P. Müller, A. A. Golubov, A. Brinkman, G. Crecilius, A. Kaluza, and H. Lüth, Phys. Rev. B 67, 014522 (2003). 